\title{
Tailoring and enhancing spontaneous two-photon emission using resonant plasmonic nanostructures
}

\author{
Alexander N. Poddubny, ${ }^{1,3}$ Pavel Ginzburg, ${ }^{2, *}$ Pavel A. Belov, ${ }^{1,4}$ Anatoly V. Zayats, ${ }^{2}$ and Yuri S. Kivshar ${ }^{1,5}$ \\ ${ }^{1}$ National University for Information Technology, Mechanics and Optics (ITMO), St. Petersburg 197101, Russia \\ ${ }^{2}$ Department of Physics, King's College London, Strand, London WC2R 2LS, United Kingdom \\ ${ }^{3}$ Ioffe Physical-Technical Institute of the Russian Academy of Sciences, St. Petersburg 194021, Russia \\ ${ }^{4}$ School of Electronic Engineering and Computer Science, Queen Mary University of London, London E1 4NS, United Kingdom \\ ${ }^{5}$ Nonlinear Physics Center and Center for Ultrahigh-bandwidth Devices for Optical Systems (CUDOS), \\ Australian National University, Canberra ACT 0200, Australia \\ (Received 7 March 2012; published 20 September 2012)
}

\begin{abstract}
The rate of spontaneous emission is known to depend on the environment of a emitter, and the enhancement of one-photon emission in a resonant cavity is known as the Purcell effect. Here we develop a theory of spontaneous two-photon emission for a general electromagnetic environment including inhomogeneous dispersive and absorptive media. This theory is used to evaluate the two-photon Purcell enhancement in the vicinity of metallic nanoparticles and demonstrates that the surface-plasmon resonances supported by these particles can increase the emission rate by more than two orders of magnitude. The control over two-photon Purcell enhancement given by tailored nanostructured environments could provide an emitter with any desired spectral response and may serve as an ultimate route for designing light sources with novel properties.
\end{abstract}

DOI: 10.1103/PhysRevA.86.033826

PACS number(s): 42.50.Nn, 42.65.Yj, 42.82.Fv, 78.67.Bf

\section{INTRODUCTION}

Spontaneous two-photon emission (STPE) is a quantum process, where an excited electron decays to its ground state by the simultaneous emission of a photon pair. It corresponds to a second-order term in perturbation series describing the system de-excitation. The phenomenon allows any combination of photon energies satisfying total energy conservation, resulting in a very broad emission spectrum. Multiphoton quantum states, and in particular, those involving just two photons, are playing key role in quantum cryptography and computing. For example, entangled state generators [1], amplifiers [2], and lasers [3] may exhibit unusual properties, such as generation of two-photon coherent states [4], quantum noise quenching and squeezing [5], and continuous variable generation, useful for quantum cryptography [6]. STPE is one of the fundamental quantum phenomena, and its applications span from information and communication technologies to biological imaging and related research [7].

STPE has recently been observed in optically pumped and electrically driven semiconductor structures [8]. STPE from semiconductors is comparable with other nonlinear processes, such as spontaneous parametric down-conversion (SPDC), and may lead to a new route for quantum devices based on nonclassical states of light [9]. Efficient, high-rate, compact, room-temperature sources of entangled photon pairs based on STPE have also been recently proposed [10].

In this paper we develop the general theoretical approach to STPE description for a pointlike emitter embedded within an arbitrary environment, emphasizing the proper treatment of dispersion and absorption of the material components. As an example, we have considered a two-photon emission near a spherical metallic nanoparticle and demonstrated that control over the emission spectrum and strong Purcell-like

*Corresponding author: pavel.ginzburg@kcl.ac.uk enhancement of the radiative decay can be achieved by tuning parameters of the palsmonic system.

\section{THEORETICAL MODEL}

Since the pioneering work of Purcell [11], it is well known that the strength of light-matter interaction can be significantly influenced by engineering the local structure of electromagnetic modes, e.g., in a cavity. When such manipulation of light emission is required in the optical spectral range, a number of different systems may be used, such as photonic crystals [12], metal nanostructures [13,14], and metamaterials [15-17]. Until recently, the majority of these systems have only been applied to one-photon processes. The Purcell factor $P_{n}$ of high-order emission processes involving $n$-photons, is of great importance, since they are initially weak and may be enhanced as approximately the nth power of the one-photon $\left(P_{1}\right)$ enhancement. The STPE from a bulk semiconductor layer interfaced with a metallic nanoantenna has recently been demonstrated to exhibit up to $10^{3}$-fold overall enhancement [18]. Although the resonant plasmonic enhancement of Raman scattering and nonlinear optical effects is well known [19], the enhancement of STPE which involves emission of photons in a broad spectral range from teraherz to visible wavelengths, has not been elaborated on yet.

The quantization of electromagnetic field in the presence of material bodies requires significant care. Material dispersion and absorption (related to each other by the Kramers-Kronig relations) increase the complexity of a canonical Hamiltonian quantization, since additional "material" degrees of freedom must be included. The easiest approach for field quantization, based on field expansion over the classical electromagnetic modes of a structure and known as mode decomposition, is not applicable to these lossy systems [20]. One of the rigorous techniques for field quantization is based on an introduction of local noise operators and results in local quantization of an 


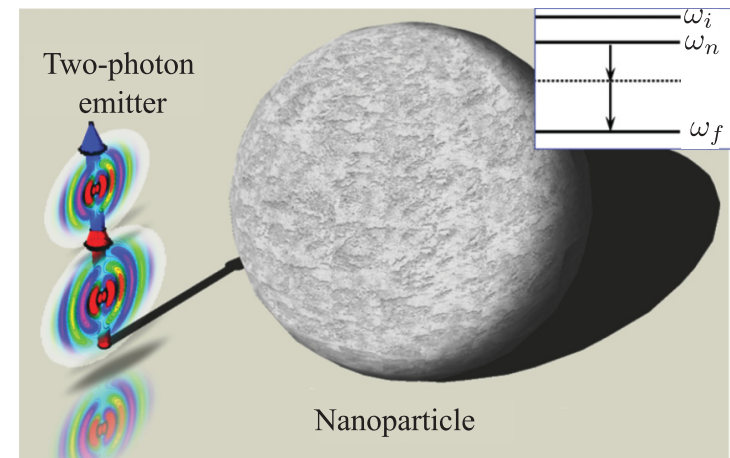

FIG. 1. (Color online) Two-photon dipole emitter positioned in the proximity to a nanoparticle. (Insert) Electronic level structure of the emitter: (solid lines) initial, final and intermediate states, (dotted line) virtual level.

electromagnetic domain. Here, the field operators are strictly related to the classical electromagnetic Green's functions [21], and the dispersion and losses can be included without violating canonical commutation rules. In this approach, the photon momentum is not directly defined, in contrast to the mode decomposition technique. However, while certain restrictions on momenta can be important for some phenomena where atomic recoil should be taken into account, in the noisy solidstate environment this is of less significance.

We consider a subwavelength light-emitting system such as an atom or a quantum dot having a discrete set of levels $\{|n\rangle\}$ and placed at the point $\vec{r}_{A}$ in the photonic environment characterized by the (dispersive and lossy) dielectric function $\varepsilon(\vec{r}, \omega)$ (e.g., Fig. 1). We restrict ourselves to the dipole transitions, characterized by the matrix elements $d_{n \rightarrow m}$, and apply a rotating wave approximation. The total Hamiltonian of the system is given by [20]

$$
\begin{aligned}
\widehat{H}= & \widehat{H}_{0}+\widehat{H}_{\text {field }}+\widehat{H}_{\text {int }}, \\
\widehat{H}_{0}= & \sum_{n} \hbar \omega_{n}|n\rangle\langle n|, \\
\widehat{H}_{\text {field }}= & \int d^{3} \vec{r} \int_{0}^{\infty} d \omega \hbar \omega \widehat{f}^{\dagger}(\vec{r}, \omega) \widehat{f}(\vec{r}, \omega), \\
\widehat{H}_{\text {int }}= & \int_{0}^{\infty} d \omega \int d^{3} \vec{r} \widehat{V}(\vec{r}, \omega), \\
\widehat{V}(\vec{r}, \omega)= & -i \sqrt{\frac{\hbar}{\pi \varepsilon_{0}}} \sum_{m, n} \int_{0}^{\infty} d \omega\left(\frac{\omega}{c}\right)^{2} \\
& \times \int d^{3} \vec{r} \sqrt{\operatorname{Im} \varepsilon(\vec{r}, \omega)} d_{n \rightarrow m} \\
& \times \overleftrightarrow{G}\left(\vec{r}_{A}, \vec{r}, \omega\right) \widehat{f}(\vec{r}, \omega) \widehat{\sigma}_{m n}+\text { H.c. },
\end{aligned}
$$

where $\widehat{H}_{0}, \widehat{H}_{\text {field }}$, and $\widehat{H}_{\text {int }}$ are the Hamiltonians of the unperturbed atomic system, free electromagnetic field, and light-matter interaction, respectively. Here, $\widehat{f}^{\dagger}(\vec{r}, \omega), \widehat{f}(\vec{r}, \omega)$ are the local creation and annihilation field operators dependent on position in space $\vec{r}$ and frequency $\omega$, and $\vec{G}\left(\vec{r}_{A}, \vec{r}, \omega\right)$ is the classical electromagnetic Green's tensor defined by

$$
\left[\nabla \times \nabla \times-\left(\frac{\omega}{c}\right)^{2} \varepsilon(\vec{r}, \omega)\right] \overleftrightarrow{G}\left(\vec{r}, \vec{r}_{A}\right)=\delta\left(\vec{r}-\vec{r}_{A}\right) .
$$

Here, $\vec{r}_{A}$ is a source position and $\vec{r}$ is an arbitrary point in space, $\widehat{\sigma}_{m n}=|m\rangle\langle n|$, and the rest of the symbols are conventional notations.

The spontaneous one-photon emission rate $\left(R_{\mathrm{SOPE}}\right)$ in the weak-coupling regime and within the framework of the local operators' formalism is given by [20]

$$
\begin{gathered}
R_{\mathrm{SOPE}}=R_{0} \frac{\vec{d}_{2 \rightarrow 1}^{*} \overleftrightarrow{P}_{1} \vec{d}_{1 \rightarrow 2}}{\left|d_{2 \rightarrow 1}^{2}\right|}, \\
\overleftrightarrow{P}_{1}\left(\vec{r}_{A}, \omega_{\mathrm{if}}\right)=\frac{3 c}{2 \omega_{\mathrm{if}}} \operatorname{Im} \stackrel{\leftrightarrow}{G}\left(\vec{r}_{A}, \vec{r}_{A}, \omega_{\mathrm{if}}\right),
\end{gathered}
$$

where $\omega_{\mathrm{if}}$ is the frequency of the transition and $R_{0}=$ $4 \omega_{\text {if }}^{3}\left|d_{2 \rightarrow 1}\right|^{2} / 3 \hbar c^{3} \varepsilon_{0}$ is the decay rate in vacuum. Here, $\overleftrightarrow{P}_{1}$ is the tensor of Purcell factor, determined by the imaginary part of the Green function. In the case of a single mode cavity with the quality factor $Q$, modal volume $V$, and wavelength $\lambda, \overleftrightarrow{P}_{1}$ is proportional to the well-known expression $Q \lambda^{3} / V$, where a crucial assumption of negligible losses in the material components is required, making this expression unsuitable for our purposes. Moreover, some nonexponential decays in plasmonic structures may show off [22].

The STPE rate for the system described by Eq. (1) has been calculated by the standard quantum-mechanical second-order perturbation theory, utilizing the local quantization scheme. The STPE rate between initial and final states $(i \rightarrow f)$ via complete set of intermediate states $\{|n\rangle\}$ is given by

$$
\begin{aligned}
R_{\text {STPE }}= & \frac{2 \pi}{\hbar} \int_{0}^{\infty} d \omega \int d^{3} \vec{r} \\
& \times \mid \sum_{n}\langle f|\widehat{V}(\vec{r}, \omega)| n\rangle\langle n|\widehat{V}(\vec{r}, \omega)| i\rangle \\
& \times\left.\left(\frac{1}{\omega_{i n}-\omega}+\frac{1}{\omega-\omega_{n f}}\right)\right|^{2} \\
& \times \delta\left(E_{i}-E_{f}-\hbar \omega\right) .
\end{aligned}
$$

Using the Green function identities [21], one can express the result via the Purcell factor, Eq. (3), as

$$
\begin{aligned}
R_{\mathrm{STPE}}= & \int_{0}^{\omega_{\mathrm{if}}} d \omega \operatorname{Tr}\left[\overleftrightarrow{P}_{1}(\omega) \overleftrightarrow{P}_{1}\left(\omega_{\mathrm{if}}-\omega\right)\right] U(\omega), \\
U(\omega)= & \frac{4 \pi \omega_{\mathrm{if}}^{3}\left(\omega_{\mathrm{if}}-\omega\right)^{3}}{9 \hbar c^{2} \varepsilon_{0}^{2}} \mid \sum_{n} d_{i \rightarrow n} d_{n \rightarrow f} \\
& \times\left.\left(\frac{1}{\omega_{i n}-\omega}+\frac{1}{\omega-\omega_{n f}}\right)\right|^{2}
\end{aligned}
$$

Here, we assume that the states $|i\rangle,|n\rangle$, and $|f\rangle$ have $s, p$ and $s$ orbital symmetries, respectively. Equation (5) has a transparent physical meaning: the STPE rate is given by the convolution of two one-photon Purcell factors, with the frequency-dependent factor sensitive to the relative energy positions of the states $|i\rangle$, $|f\rangle$, and $\{|n\rangle\}$. The difference between one- and two-photon Purcell effects is summarized in the schematic drawing in Fig. 2. While the one-photon emission is enhanced only at the resonance of the structure, STPE, being naturally wideband, is enhanced twice: at the resonant frequency $\left(\omega_{\text {res }}\right)$ of a structure and at the complementary one $\left(\omega_{\text {if }}-\omega_{\text {res }}\right)$, 


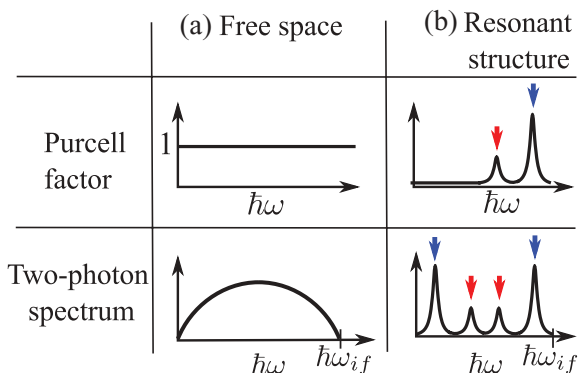

FIG. 2. (Color online) One-photon Purcell factor and two-photon spectra in a free space and near resonant structure with two resonances. Wideband STPE is enhanced twice: at the resonant frequencies $\left(\omega_{\text {res }}\right)$ and the complimentary frequencies $\left(\omega_{\text {if }}-\omega_{\text {res }}\right)$.

emphasizing the "paired" nature of this energy-time entangled emission process.

Additional radiative and nonradiative decay channels, leading to the broadening of the energy levels, may be taken into account in Eqs. (4) and (5) phenomenologically, by replacing the denominators with $\omega_{\text {in }}-\omega+i \gamma$ and $\omega-\omega_{n f}+$ $i \gamma$, respectively. The broadening $\gamma$ is related to the spectral shape $\Gamma(\omega)$, which may be calculated for each physical system, e.g., it has a Lorentzian shape for radiative broadening in a collisionless dilute gas, but will be more complicated for other systems, such as solid-state emitters [23]. The modified version of Eq. (5) for the spectral density, including these effects is

$$
\bar{U}(\omega)=\int d \omega U(\omega, \gamma(\omega)) \Gamma(\omega) .
$$

In the more general case different values of broadening should be used for each of the states $|i\rangle,|f\rangle$, and $\{|n\rangle\}$. In what follows we assume $\Gamma(\omega)=\delta(\omega)$ for simplicity.

It is important to assess the role of losses in the medium, determined by the imaginary part of the dielectric constant. Only some of the emitted photons contributing to the true radiative decay may be observed in the far field, while the rest are absorbed by the lossy medium [24]. The specific advantage of the Green function technique we use [21] is the possibility to straightforwardly single out the radiative part of the Purcell factor:

$$
\begin{aligned}
\stackrel{\leftrightarrow}{P}_{1, \mathrm{RAD}}\left(\vec{r}_{A}, \omega_{\mathrm{if}}\right)= & \stackrel{\leftrightarrow}{P}_{1}\left(\vec{r}_{A}, \omega_{\mathrm{if}}\right)-\frac{3 \omega_{\mathrm{if}}}{2 c} \int d^{3} \vec{r} \operatorname{Im} \varepsilon(\vec{r}) \\
& \times \stackrel{\leftrightarrow}{G}^{*}\left(\vec{r}_{A}, \vec{r}, \omega_{\mathrm{if}}\right) \stackrel{\leftrightarrow}{G}\left(\vec{r}, \vec{r}_{A}, \omega_{\text {if }}\right)
\end{aligned}
$$

where the second term corresponds to the Joule losses. Substituting Purcell factor (7) in Eq. (5), we obtain the "true" radiative STPE decay rate. Equations (3), (5), (7), and Fig. 2 provide general concept for two-photon emission engineering. By properly tailoring the nanostructured environment utilizing plasmonic, dielectric, or other resonances, one can obtain a system with a desired emission spectrum.

\section{NUMERICAL EXAMPLE}

In the following, for the sake of simplicity, we will consider a specific case of a three-level atom near a nanosphere (Fig. 1). We consider a single intermediate state with the energy above both initial and final states (Fig. 1, inset) and neglect line

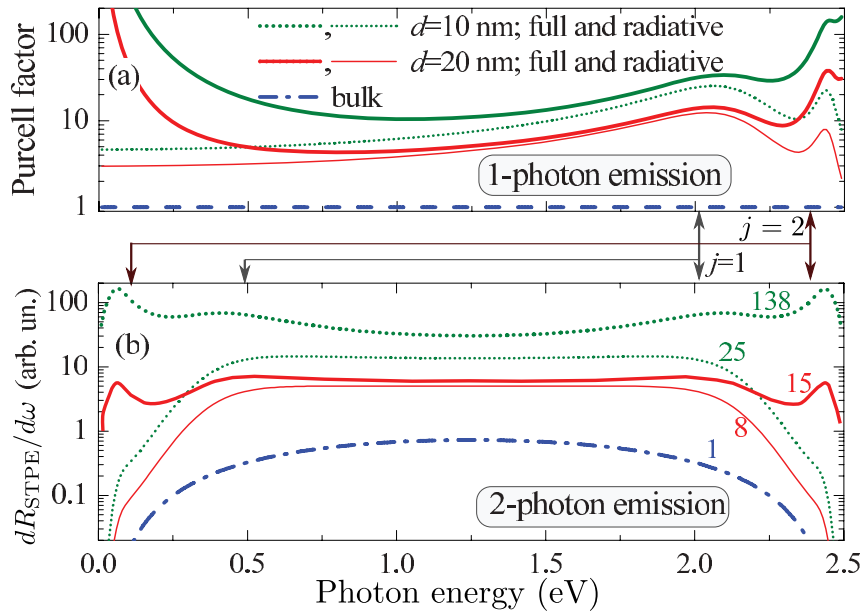

FIG. 3. (Color online) (a) The Purcell factor for a dipole oriented perpendicular to an $\mathrm{Ag}$ sphere at various distances from its surface. The thick and thin lines correspond to the full Purcell factor, Eq. (3), and its radiative part, Eq. (7), respectively. (b) The spectral densities of STPE normalized to the overall emission rate in an empty silica matrix (i.e., the area under the blue-dashed line is unity). Numbers near the curves show the integrated over the whole spectral range STPE enhancement with respect to the bulk $\mathrm{SiO}_{2}$ matrix. Thick and thin lines correspond to the total STPE rate and its radiative part, respectively. Vertical arrows indicate the energies of the electric multipole modes with different total angular momentum values $j . d$ is the distance between the emitter in $\mathrm{SiO}_{2}$ matrix and the nanoparticle surface and indicated in the figure. Nanoparticle diameter is $100 \mathrm{~nm}$. The transition energy $\omega_{\text {if }}=2.5 \mathrm{eV}$.

broadening. To examine the effect of local field enhancement, we have analyzed the case when the emitter is placed in $\mathrm{a} i O_{2}$ matrix in the proximity of both silicon and silver spheres of the same subwavelength dimensions. Metallic nanoparticles with negative permittivity may support collective oscillations of surface charges in the optical and infrared spectral range termed as localized surface-plasmon resonances (LSP) [25]. Such plasmonic particles can concentrate the optical field beyond the classical diffraction limit [26], while the resonant response of the dielectric particles with positive permittivity (such as Si) may only result from wave interference, and hence is not possible at subwavelength dimensions. For the chosen geometry the electrodynamical Green function can be readily found as series over spherical harmonics. The dielectric function of $\mathrm{Si}$ and the Drude parameters of $\mathrm{Ag}$ were taken from widely available experimental data, while the value $\varepsilon=2$ was chosen for the permittivity of $\mathrm{SiO}_{2}$.

Figure 3 presents the spontaneous one- and two-photon emission spectra for a source located at different distances $d$ from the surface of the Ag particle. Panel (a) shows the calculated energy dependence of the one-photon Purcell factor [Eq. (3)] for a dipole oriented along the line between emitter and nanoparticle center. The peak positions correspond to LSP resonances of dipolar $(j=1)$ and quadrupolar $(j=2)$ nature. The Purcell factor at low frequencies [thick lines in panel (a)] has a nonradiative origin and is related to the Joule heating of the metal. This maximum vanishes for the radiative part of the Purcell factor Eq. (6), shown by thin lines. 
Figure 3(b) presents the normalized spectral dependence of STPE. These graphs possess characteristic mirror symmetry with respect to the central energy $\hbar \omega_{\text {if }} / 2$. The blue-dashed curve in Fig. 3(b) corresponds to the STPE spectrum for an infinite distance $d$ between the atom and the nanoparticle, i.e., an emitter embedded in an empty silica matrix. In this case, the STPE spectrum is smooth and depends only on the "free-spacelike" photon density of states $\left(\sim \omega^{2}\right)$. The highest emission probability corresponds to the degenerate case (equal energies of the emitted photons), so that the product of their densities of states is at a maximum [Eqs. (4) and (5)]. Figure 3 shows a growth in the emission rate with the decrease of the distance between the emitter and the sphere. In the case of the $\mathrm{Ag}$ nanoparticle, the overall integrated enhancement of STPE at $d=10 \mathrm{~nm}$ is about 140 -fold.

The STPE spectrum exhibits a rich multipeak structure due to the plasmon resonances. This resonant structure can be most easily understood when compared with that in the one-photon spectra [Fig. 3(a)]. The two central maxima in Fig. 3(b), marked by the arrows: $j=1$ corresponds to the electric dipole resonance leading to the emission enhancement at its frequency $\omega_{d}$ and the complementary frequency $\left(\omega_{\mathrm{if}}-\omega_{d}\right)$. The bordering peaks are related to higher-order multipole resonances. One can see from Fig. 3(b) that the higherorder resonances become more pronounced when the emitter approaches the nanoparticle since the multipole modes have stronger field localization near the surface. The transition energy $\hbar \omega_{\text {if }}$ in Fig. 3 was chosen to be slightly above the dipole and quadrupole plasmonic resonances of the nanoparticle, lying in the optical spectral range [Fig 3(a)]. As a result, the counterparts of these plasmonic resonances manifest themselves in the STPE in the IR and THz ranges. This possibility to "convert" structure resonances from high to low frequencies (and vice versa) [32] along with recent advances in the engineering of the spectral response of plasmonic particles [27-31] reveals the potential ability to design STPE spectra for specific applications.

The thin curves in Fig. 3(b) show the far-field radiative contribution to the STPE rate, where Joule losses are subtracted. A comparison between thick and thin lines reveals the significant role of the Joule losses for low- and high-energy ranges of the STPE spectrum [Fig. 3(a)]. Nevertheless, the overall radiative STPE rate, as well the rate of emission of $\mathrm{THz}$ photons, is still enhanced when compared to that in bulk $\mathrm{SiO}_{2}$ matrix.

Results in Fig. 3 directly show the advantage of the microscopic Green function technique used here over the approximate phenomenological Lorentzian cavity approach [18]. First, the maxima in Purcell factor, Fig. 3(a), are strongly broadened and hence substantially non-Lorentzian. Second, the theory of Ref. [18] does not allow one to take into account the Joule losses, which strongly affect both the absolute value of emission rate enhancement [Fig. 3(b)] and the (non-Lorentzian) spectral shape [Fig. 3(a)]. The simplified approach may still be valid for specific structures with single-peak Lorentzian Purcell enhancement spectrum, such as plasmonic bow-tie antennas of Ref. [18], but the only general way to describe lossy nanoparticles with multiple plasmonic resonances is the Green function technique.

It is also instructive to analyze the dependence of STPE on the spectral position of the plasmonic resonance with respect to

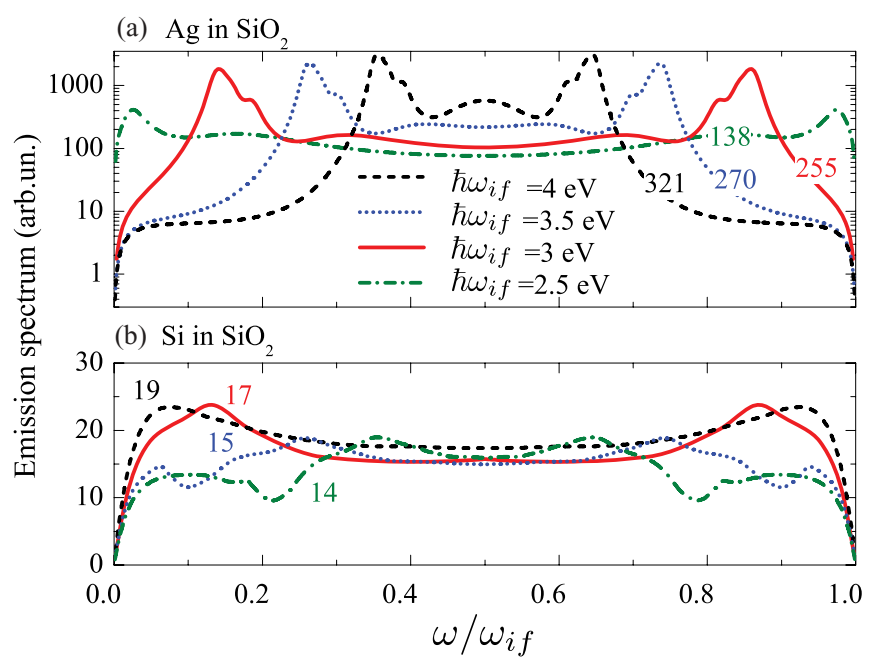

FIG. 4. (Color online) Normalized STPE spectra calculated for different transition energies $\hbar \omega_{\mathrm{if}}$ and the fixed LSP resonances: (a) and (b) correspond to $\mathrm{Ag}$ and $\mathrm{Si}$ nanoparticles in $\mathrm{SiO}_{2}$ matrix, respectively. The photon energy on the $x$ axes is normalized to the transition energy. Calculation parameters and notations are the same as in Fig. 3.

the emission frequency (Fig. 4). The spectra calculated for both $\mathrm{Ag}$ and Si particles for different transition energies $\hbar \omega_{\text {if }}$ with fixed energy of the intermediate state $\hbar \omega_{\mathrm{ni}}=0.3 \mathrm{eV}$, are presented in Fig 4. All of the graphs are normalized to the overall emission rate in the empty silica matrix. For the Si nanoparticle, the spectral dependencies are relatively smooth and structureless; the enhancement results from the density of states near the high-index dielectric boundary [Fig. 4(b)]. The overall enhancement weakly depends on the transition energy. On the other hand, the plasmonic particle allows further improvement of the emission rate by a factor of up to 320 . The origin of this effect is the spectral overlap between the two resonances at $\omega_{d}$ and $\omega_{\text {if }}-\omega_{d}$. This may be further increased by placing the emitter even closer to the interface. Figure 5 shows the radiative contribution to the STPE emission rate calculated for

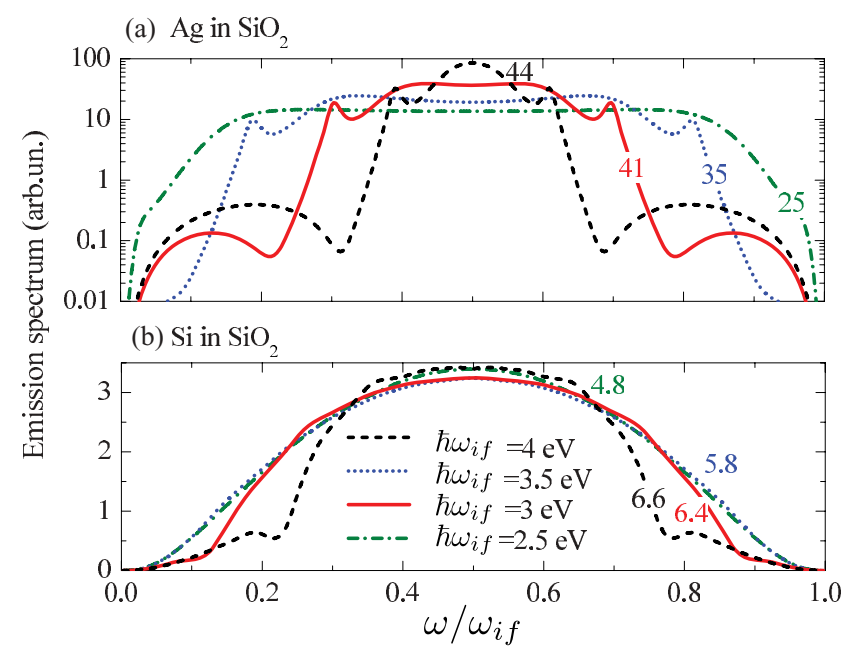

FIG. 5. (Color online) The radiative part of the normalized STPE spectra calculated for different transition energies $\hbar \omega_{\text {if }}$ and the fixed LSP resonances. Notations and calculation parameters are the same as in Fig. 4. 
the parameters of Fig. 4. By comparing Figs. 5 and 4, one can see that despite a considerable reduction of the far-field STPE intensity due to the losses, the effect of plasmonic resonances remains significant and gives rise to a complex STPE spectrum.

These examples demonstrate that the simplest spherical nanoparticle can dramatically modify the characteristic STPE properties of an emitter. A proper design of multiresonant plasmonic structures with variable and tunable quality factors may, in principle, provide any spectral response on demand. Moreover, in the STPE process, the emitted photons are always time-energy entangled and thus, such a multiresonant plasmonic environment engenders the possibility to achieve multiparticle entanglement. This problem requires separate studies since the entanglement depends not only on the plasmonic environment, but also on the specific choice of the quantum light source and on the excitation mechanism [33].

\section{CONCLUSIONS}

In conclusion, we have developed a rigorous analytical approach to evaluate spontaneous two-photon emission rates in a structured metallodielectric photonic environment. The local field operators enable the formulation of this purely quantum problem in terms of classical electrodynamics by expressing the two-photon emission spectrum via the one-photon Purcell factors. As an example, the influence of localized surface-plasmon resonances on the fundamental multiphoton processes was demonstrated by the full analytical calculation of emission rates in the vicinity of nanoscale particles. The unique nature of localized surface plasmons to confine light on subwavelength scales leads to at least two to three orders of magnitude enhancement of the emission together with on-demand spectral reshaping in a broad spectral range.

\section{ACKNOWLEDGMENTS}

This work was supported in part by the EPSRC (UK) and Australian Research Council (Australia). A.P. acknowledges support from RFBR and the European projects POLAPHEN and Spin-Optronics. P.G. acknowledges the Royal Society for support from a Newton International Fellowship and the Yad Hanadiv Foundation for support from a Rothschild Fellowship. A. Poddubny also acknowledges fruitful discussions with M. M. Glazov.
[1] P. G. Kwiat, K. Mattle, H. Weintfurter, A. Zeilinger, A. V. Sergienko, and Y. Shih, Phys. Rev. Lett. 75, 4337 (1995).

[2] A. Nevet, A. Hayat, and M. Orenstein, Phys. Rev. Lett. 104, 207404 (2010).

[3] D. J. Gauthier, Q. Wu, S. E. Morin, and T. W. Mossberg, Phys. Rev. Lett. 68, 464 (1992).

[4] H. P. Yuen, Phys. Rev. A 13, 2226 (1976).

[5] M. O. Scully, K. Wodkiewicz, M. S. Zubairy, J. Bergou, N. Lu, and J. Meyer ter Vehn, Phys. Rev. Lett. 60, 1832 (1988).

[6] F. Grosshans and P. Grangier, Phys. Rev. Lett. 88, 057902 (2002).

[7] M. B. Nasr, D. P. Goode, N. Nguyen, G. Rong, L. Yang, B. M. Reinhard, B. E. A. Saleh, and M. C. Teich, Opt. Commun. 282, 1154 (2009).

[8] A. Hayat, P. Ginzburg, and M. Orenstein, Nature Photon. 2, 238 (2008).

[9] H. M. van Driel, Nature Photon. 2, 212 (2008).

[10] A. Hayat, P. Ginzburg, and M. Orenstein, Phys. Rev. B 76, 035339 (2007).

[11] E. M. Purcell, Phys. Rev. 69, 681 (1946).

[12] P. Lodahl, A. F. van Driel, I. S. Nikolaev, A. Irman, K. Overgaag, D. Vanmaekelbergh, and W. L. Vos, Nature (London) 430, 654 (2004).

[13] L. A. Blanco and F. J. García de Abajo, Phys. Rev. B 69, 205414 (2004).

[14] J. B. Khurgin, G. Sun, and R. A. Soref, J. Opt. Soc. Am. B 24, 1968 (2007).

[15] Z. Jacob, J. Kim, G. V. Naik, A. Boltasseva, E. E. Narimanov, and V. M. Shalaev, Appl. Phys. B 100, 215 (2010).

[16] K. Tanaka, E. Plum, J. Y. Ou, T. Uchino, and N. I. Zheludev, Phys. Rev. Lett. 105, 227403 (2010).
[17] A. N. Poddubny, P. A. Belov, and Y. S. Kivshar, Phys. Rev. A 84, 023807 (2011).

[18] A. Nevet, N. Berkovitch, A. Hayat, P. Ginzburg, S. Ginzach, O. Sorias, and M. Orenstein, Nano Lett. 10, 1848 (2010).

[19] M. Moskovits, Rev. Mod. Phys. 57, 783 (1985).

[20] N. A. R. Bhat and J.E. Sipe, Phys. Rev. A 73, 063808 (2006).

[21] W. Vogel and D. Welsch, Quantum Optics (Wiley-VCH, Weinheim, 2006).

[22] P. Ginzburg and A. V. Zayats, Opt. Express 20, 6720 (2012).

[23] A. Hayat, P. Ginzburg, and M. Orenstein, Phys. Rev. Lett. 103, 023601 (2009).

[24] M. M. Glazov, E. L. Ivchenko, A. N. Poddubny, and G. Khitrova, Phys. Solid State 53, 1753 (2011).

[25] S. A. Maier, Plasmonics: Fundamentals and Applications (Springer, New York, 2007).

[26] D. K. Gramotnev and S. I. Bozhevolnyi, Nature Photon. 4, 83 (2010)

[27] N. Berkovitch, P. Ginzburg, and M. Orenstein, J. Phys.: Condens. Matter 24, 073202 (2012).

[28] E. Prodan, C. Radloff, N. J. Halas, and P. Nordlander, Science 302, 419 (2003).

[29] P. Ginzburg, N. Berkovitch, A. Nevet, I. Shor, and M. Orenstein, Nano Lett. 11, 2329 (2011).

[30] V. Giannini and J. A. Sánchez-Gil, Opt. Lett. 33, 899 (2008).

[31] H. Harutyunyan, G. Volpe, R. Quidant, and L. Novotny, Phys. Rev. Lett. 108, 217403 (2012).

[32] A. V. Kavokin, I. A. Shelykh, T. Taylor, and M. M. Glazov, Phys. Rev. Lett. 108, 197401 (2012).

[33] A. N. Poddubny, Phys. Rev. B 85, 075311 (2012). 